\title{
Diagnostic accuracy of urine neutrophil gelatinase-associated lipocalin and urine kidney injury molecule-1 as predictors of acute pyelonephritis in young children with febrile urinary tract infection
}

\author{
GRAŻYNA KRZEMIEN' ${ }^{1}$, MAŁGORZATA PAŃCZYK-TOMASZEWSKA', IWONA KOTUŁA2, \\ URSZULA DEMKOW' ${ }^{2}$ AGNIESZKA SZMIGIELSKA ${ }^{l}$ \\ ${ }^{1}$ Department of Pediatrics and Nephrology, Medical University of Warsaw, Poland \\ ${ }^{2}$ Department of Laboratory Diagnostics and Clinical Immunology of Developmental Age, Medical University of Warsaw, Poland
}

\begin{abstract}
Introduction: We assessed whether two urinary biomarkers of acute kidney injury, neutrophil gelatinase associated lipocalin ( $U N G A L)$ and kidney injury molecule-1 (uKIM-1), can be useful for predicting acute pyelonephritis (APN) in children aged 1-24 months with the first febrile urinary tract infection (UTI).

Material and methods: A prospective study included 54 children divided into two groups (24 with $A P N, 30$ with lower UTI), according to the dimercaptosuccinic acid (DMSA) renal scintigraphy results. Laboratory tests: uNGAL, uKIM-1, procalcitonin (PCT), C-reactive protein $(C R P)$, white blood count (WBC) were performed.

Results: We did not find significant differences in normalized and non-normalized values of uNGAL and UKIM-1 in children with APN and lower UTI. Positive correlations were determined between $u N G A L$ and pyuria $(r=0.28, p<0.05)$ and between $u N G A L / u C r$ and $u K I M-1 / u C r(r=0.53, p<0.001)$ in the all UTI groups. Univariate logistic regression analysis demonstrated that only PCT $(p<0.0001)$ and CRP $(p<0.05)$ were important diagnostic factors of APN. Receiver operating curve $(R O C)$ analysis showed good diagnostic profiles of PCT with the best cut-off value of $1.66 \mathrm{ng} / \mathrm{ml}$ and of CRP with the best cut-off value of $4.3 \mathrm{mg} / \mathrm{dl}$ for predicting APN (area under the curve [AUC]: 0.894 and 0.719 , sensitivity: $75 \%$ and $96 \%$, specificity: $93 \%$ and $43 \%$, respectively).

Conclusions: $U N G A L$ and $U K I M-1$ are not effective diagnostic markers for APN in young children with febrile UTI and cannot be used in clinical practice to differentiate APN from lower UTI.
\end{abstract}

Key words: acute pyelonephritis, urinary tract infection, acute kidney injury, biomarkers, neutrophil gelatinase-associated lipocalin, kidney injury molecule-1, dimercaptosuccinic acid scintigraphy, children.

(Centr Eur J Immunol 2019; 44 (2): 174-180)

\section{Introduction}

Urinary tract infection (UTI) is regarded as one of the most prevalent bacterial infections in the early life, afflicting 5-15.6\% of children aged 1-24 months presenting with fever [1-3]. Young children with UTI are more likely than older children to have acute pyelonephritis (APN) [4]. In clinical practice, fever along with acute-phase inflammatory markers has been used to differentiate between APN and lower UTI [5, 6]. However, these clinical and labora- tory symptoms do not accurately localize APN and lower UTI $[4,7]$. 99mTc-dimercaptosuccinic acid (DMSA) renal scintigraphy, a gold standard for detecting APN, has wellknown limitations, and the routine use of DMSA scan for localization of UTI is not recommended [1, 8]. Early diagnosis of APN in young children is essential to prevent or reduce long-term renal sequels [8,9]. Therefore, it is important to find noninvasive methods for detecting APN. Several biomarkers have been investigated for UTI and APN diagnosis in children and adults [10-12].

Correspondence: Agnieszka Szmigielska, MD, PhD, Department of Pediatrics and Nephrology, Medical University of Warsaw, 63 Żwirki i Wigury St., 02-091 Warsaw, Poland, e-mail: agnieszka.szmigielska@wum.edu.pl Submitted: 28.02.2019; Accepted: 17.04.2019 
Neutrophil gelatinase-associated lipocalin (NGAL) and kidney injury molecule-1 (KIM-1) were initially evaluated in patients with acute kidney injury (AKI) $[13,14]$. NGAL, a member of the lipocalin family, plays an important role in the innate immune response to the bacterial infection $[15,16]$. In AKI it is massively expressed in the distal part of the nephron $[13,17]$. KIM-1, a type-1 transmembrane glycoprotein, is highly expressed in proximal tubule cells after ischemic or toxic AKI [18]. Numerous studies have demonstrated usefulness of both biomarkers for predicting and monitoring AKI of diverse etiologies [3, 13, 19, 20]. Recent data show also utility of NGAL and KIM-1 for detecting UTI [12, 21-23] and APN [4, 14, 18, 24] in children and adults. The studies published so far are small in size $[5,6,21,25]$ and results are contentious [26-28]. The aim of this study was to evaluate the usefulness of uNGAL and uKIM-1 for predicting APN in children aged 1-24 months with febrile UTI.

\section{Material and methods}

This prospective single-center study was performed in the Department of Pediatrics and Nephrology, between May 2016 and November 2018. The study was approved by the local Bioethics Committee for Human Research. Before enrollment, written informed consent was obtained from all parents. Fifty four children (age range: 1-24 months) with the first episode of febrile UTI were enrolled in the study. Patients with other infectious diseases or known urogenital malformations were excluded. Children were investigated with DMSA renal scan within 7-10 days after admission to the hospital and divided into APN or lower UTI group, according to DMSA results. APN was defined as focal, multifocal or diffusely decreased or absent cortical uptake, without cortical volume loss in which the renal cortical contour remains intact [8]. At the time of admission, urinalysis, urine culture, serum creatinine $(\mathrm{Cr})$, procalcitonin (PCT), C-reactive protein (CRP), and white blood cell (WBC) were performed. Urine samples were collected by bladder catheterization or midstream clean catch. Pyuria was defined as $>5 \mathrm{WBC}$ per high power field (HPF) in the urine sediment, and significant bacteriuria as growth of a single pathogen $>100,000$ colony-forming units (CFU)/ml. The UTI was recognized on the basis of presence of both pyuria and significant bacteriuria [1]. Normal values of evaluated indices in the local laboratory were as follows: $\mathrm{Cr} \leq 0.4 \mathrm{mg} / \mathrm{dl}$, PCT $<0.05 \mathrm{ng} / \mathrm{ml}$, $\mathrm{CRP} \leq 1.0 \mathrm{mg} / \mathrm{dl}$. Leukocytosis was defined as WBC count more than normal value according to age. Fever was defined as temperature of at least $38.0^{\circ} \mathrm{C}$. Urine samples for measurement of uNGAL, KIM-1 and urine $\mathrm{Cr}(\mathrm{uCr})$ were collected on the first day of hospitalization prior to antibiotic treatment. They were immediately centrifuged and stored at $-80^{\circ} \mathrm{C}$ until further analysis. uNGAL levels were measured by commercially available enzyme-linked immunosorbent assay (ELISA) kit (Human Lipocalin-2/NGAL; Cat no: RD 191102200R; Bio
Vendor Laboratory Medicine, Inc. Brno, Czech Republic) and uKIM-1 by commercially available ELISA kit (Human Kim-1; Cat no: 201-12-1100; SunRed, Shanghai, China) according to the manufacturer's instructions. The detection range for $\mathrm{uN}$ GAL was $0.3-10 \mathrm{ng} / \mathrm{ml}$ and for uKIM-1 was $0.1-10 \mathrm{ng} / \mathrm{ml}$. To exclude effects based on urinary dilution, $\mathrm{uCr}$ was used to normalize the values of biomarkers. uNGAL to $\mathrm{uCr}$ ratio (uNGAL/uCr) and uKIM-1 to uCr ratio (uKIM-1/uCr) were calculated and expressed as ng/mg. All children underwent kidney and urinary tract ultrasound (US) examination after admission to the hospital, while voiding cystourethrography (VCUG) was optional in patients with abnormal findings on US or atypical UTI.

\section{Statistical analysis}

Statistical analysis was performed using commercial Statistica package vs. 11.0 for Windows (StatSoft; Tulsa, OK). The distribution of continuous variables was tested for normality. Continuous data were presented as the mean \pm standard deviation (SD) or median with corresponding $25^{\text {th }}$ and $75^{\text {th }}$ percentiles (IQR). Categorical data were presented as numbers and percentiles. Statistical analysis of differences between two groups with normal distribution was determined by parametric Student's $t$-test. Non-parametric Mann-Whitney $U$ test was used for the variables that were not distributed normally. To compare categorical variables between two groups, the $\chi^{2}$ test was used. Correlations between variables were evaluated by linear regression analysis or Spearman's rank correlation. Univariate and multivariate logistic regression analysis was performed including $95 \%$ confidence interval (CI) to identify clinical variables associated with the presence of APN and to find an independent predictor of APN. Receiver operating characteristic (ROC) analysis was employed to calculate the area under the curve (AUC) to determine the best cut-off values, sensitivity and specificity for clinical variables to predict APN. The results were evaluated within 95\% CI. $p$ values less than 0.05 were considered statistically significant for all tests.

\section{Results}

We evaluated 54 children ( 21 boys, 33 girls) aged 1 - 24 months (mean age $7.41 \pm 4.78$ months) with the first episode of febrile UTI. Fifty $(92.6 \%)$ children were aged 1-12 months, and 4 (7.4\%) were older than 12 months. According to DMSA results, 24 (44\%) children were included in the APN group, and $30(56 \%)$ in the lower UTI group. Demographic and laboratory data of children are summarized in Table 1 . There were no significant differences in age, gender distribution and duration of fever in children with APN and lower UTI. No significant differences in UNGAL and uKIM-1 levels, as well in uNGAL/uCr and uKIM-1/uCr ratio were documented between children with APN and 
Table 1. Demographic and laboratory data of children with acute pyelonephritis and lower urinary tract infection

\begin{tabular}{|c|c|c|c|}
\hline \multirow[t]{2}{*}{ Variables } & \multicolumn{2}{|c|}{ Group } & \multirow[t]{2}{*}{$p$} \\
\hline & $\begin{array}{c}\text { APN } \\
n=24\end{array}$ & $\begin{array}{c}\text { Lower UTI } \\
n=30\end{array}$ & \\
\hline Age (months) & $7.27 \pm 4.85$ & $7.52 \pm 4.79$ & $\mathrm{~ns}$ \\
\hline Male, $n(\%)$ & $9(37.5)$ & $12(40 \%)$ & ns \\
\hline Fever duration (hours) & $66.54 \pm 55.25$ & $61.40 \pm 36.53$ & $\mathrm{~ns}$ \\
\hline uNGAL (ng/ml) & $62.98 \pm 29.37$ & $65.26 \pm 39.10$ & $\mathrm{~ns}$ \\
\hline uNGAL/uCr (ng/mg) & $168.00(85.54-295.10)$ & $209.45(110.10-332.30)$ & $\mathrm{ns}$ \\
\hline uKIM-1 (ng/ml) & $2.07 \pm 0.52$ & $2.07 \pm 0.43$ & ns \\
\hline $\mathrm{uKIM}-1 / \mathrm{uCr}(\mathrm{ng} / \mathrm{mg})$ & $5.53(2.87-11.93)$ & $7.23(3.99-12.68)$ & $\mathrm{ns}$ \\
\hline $\mathrm{PCT}(\mathrm{ng} / \mathrm{ml})$ & $4.48(1.46-7.19)$ & $0.20(0.10-0.47)$ & $<0.0001$ \\
\hline $\mathrm{CRP}(\mathrm{mg} / \mathrm{dl})$ & $13.30(6.40-16.60)$ & $5.65(2.80-9.00)$ & $<0.01$ \\
\hline $\mathrm{WBC}\left(\mathrm{mm}^{3}\right)$ & $19.71 \pm 6.87$ & $19.97 \pm 6.65$ & $\mathrm{~ns}$ \\
\hline Pyuria (uWBC/HPF) & $100(40-100)$ & $60(30-100)$ & $\mathrm{ns}$ \\
\hline
\end{tabular}
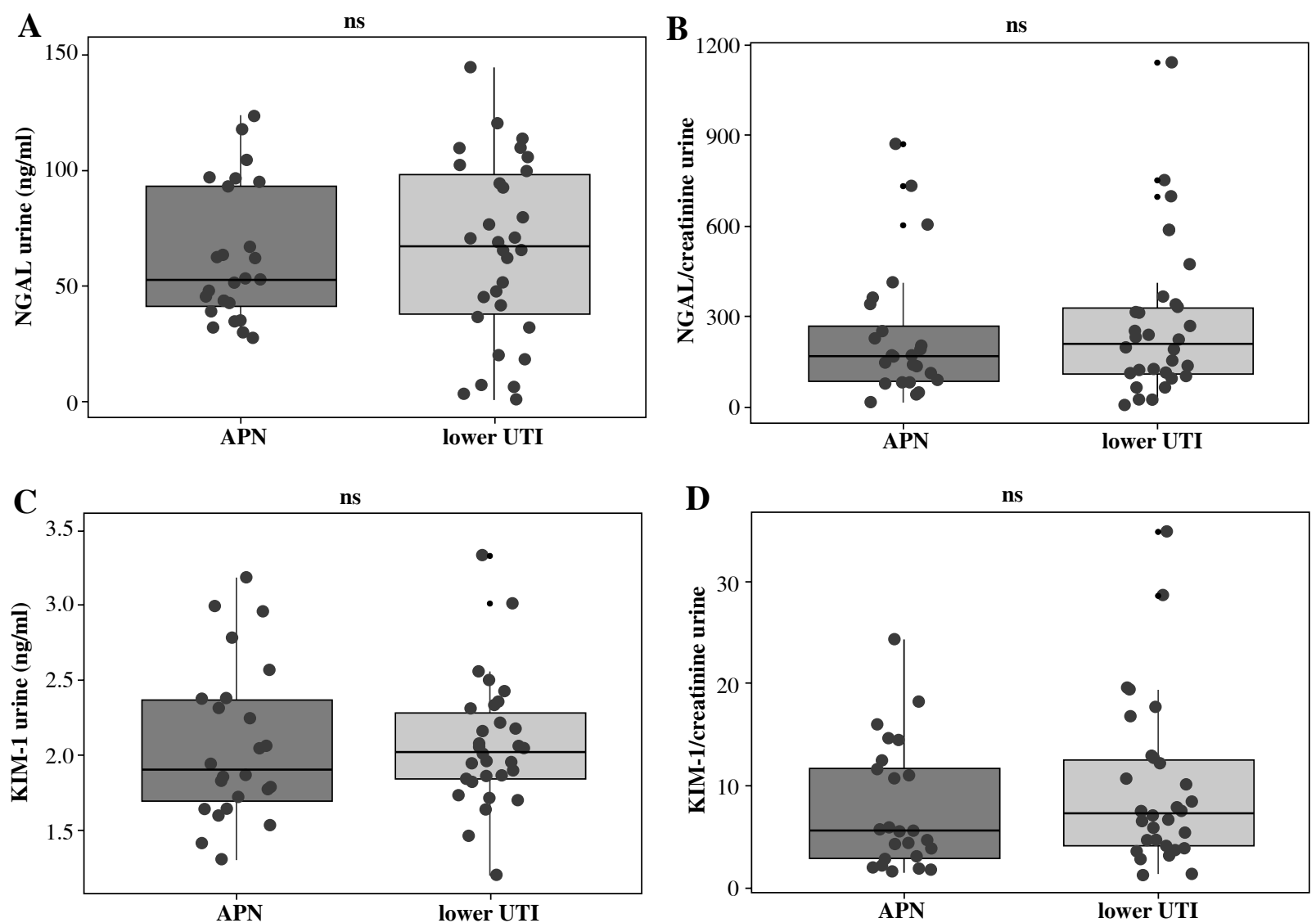

Horizontal lines represent the medians; boxes represent the interquartile range (25-75\%), whiskers above and below the box indicate the $90^{\text {th }}$ and $10^{\text {th }}$ percentiles, grey dots indicate distribution of patients, outliers are depicted as black dots.

Fig. 1. Levels of uNGAL, uNGAL normalized to $\mathrm{uCr}$ (uNGAL/uCr), uKIM-1 and uKIM-1 normalized to uCr (uKIM-1/uCr) in infants with acute pyelonephritis (APN) and lower urinary tract infection (UTI) 
lower UTI (Table 1, Fig. 1). Median PCT and mean CRP levels were significantly higher in the APN group compared to those in the lower UTI group. WBC count and pyuria did not differ between the groups. Serum levels of $\mathrm{Cr}$ were normal in all children evaluated in the study (data not shown). Escherichia coli was isolated in 52 (96.3\%) children, Klebsiella oxytoca in one child, and Proteus mirabilis in one child. Based on renal US and VCUG, mild hydronephrosis was diagnosed in $2(3.7 \%)$ children and vesicoureteral reflux grade 2 in $6(11.1 \%)$ children.

Positive correlations were found between UNGAL and pyuria $(r=0.28, p<0.05)$ and between $\mathrm{uNGAL} / \mathrm{uCr}$ and uKIM-1/uCr $(r=0.53, p<0.001)$ in the all UTI groups. Univariate logistic regression analysis has demonstrated that only PCT and CRP were important diagnostic factors of APN in children (Table 2). Multivariate analysis did not identify independent predictors of APN (data not shown). Also, ROC analysis has demonstrated that only PCT and CRP had good diagnostic profiles for predicting APN. The AUC for PCT was 0.894 (95\% CI 0.688-0.951), with the best cut-off value of $1.66 \mathrm{ng} / \mathrm{ml}$ (sensitivity $75 \%$, specificity 93\%), and the AUC for CRP was 0.719 (95\% CI 0.724-0.958), with the best cut-off value of $4.3 \mathrm{mg} / \mathrm{dl}$ (sensitivity 96\%, specificity 43\%) (Fig. 2).

\section{Discussion}

Based on the results of our study we did not find any significant differences in levels of UNGAL and UKIM-1 in children aged 1-24 months with APN and lower UTI. We determined both normalized and non-normalized values of uNGAL and UKIM-1, but normalization did not improve the results of this research. ROC analysis has demonstrated good diagnostic profiles only for PCT and CRP for predicting APN.

In 2009, Ichino et al., in an experimental rat UTI model, found a marked increase in UNGAL levels soon after the

Table 2. Univariate logistic regression analysis to identify risk factors associated with the presence of acute pyelonephritis

\begin{tabular}{lcl}
\hline Variable & OR $(\mathbf{9 5 \%} \mathbf{C I})$ & $\boldsymbol{p}$ \\
\hline Age & $0.989(0.882-1.109)$ & $\mathrm{ns}$ \\
\hline Fever duration & $1.003(0.991-1.015)$ & $\mathrm{ns}$ \\
\hline uNGAL & $0.998(0.983-1.014)$ & $\mathrm{ns}$ \\
\hline uNGAL/uCr & $0.999(0.997-1002)$ & $\mathrm{ns}$ \\
\hline uKIM-1 & $1.013(0.319-3.222)$ & $\mathrm{ns}$ \\
\hline uKIM-1/uCr & $0.962(0.888-1.043)$ & $\mathrm{ns}$ \\
\hline PCT & $4.347(1.595-11.850)$ & $<0.001$ \\
\hline CRP & $1.119(1.028-1.218)$ & $<0.05$ \\
\hline WBC & $0.994(0.917-1.078)$ & $\mathrm{ns}$ \\
\hline Pyuria & $0.998(0.992-1.024)$ & $\mathrm{ns}$
\end{tabular}

$\overline{O R}$-odds ratio, CI - confidence interval, uNGAL - urine neutrophil gelatinaseassociated lipocalin, uKIM-1 - urine kidney injury molecule-1, $u C r$ - urine creatinine, $P C T$ - procalcitonin, $C R P-C$-reactive protein, $W B C-$ white blood cell count
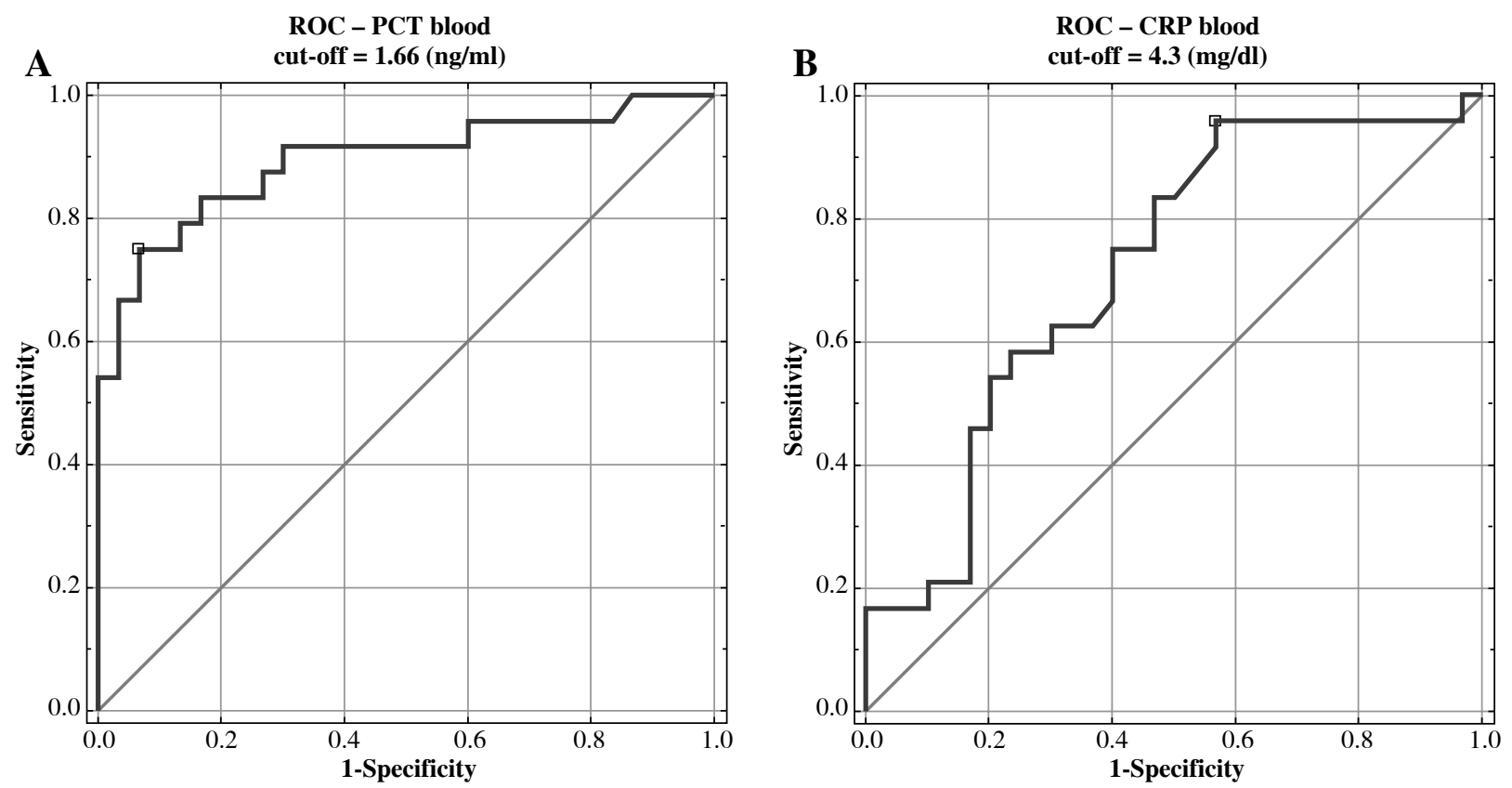

Fig. 2. Receiver operating curve to predict acute pyelonephritis (APN) for procalcitonin (PCT) and C-reactive protein (CRP) 
bacterial injection [29]. In 2013, Lee et al., in a rat APN model, demonstrated significantly elevated UNGAL and uKIM-1 levels in the acute phase of UTI. Authors suggested that both markers could be used for detection of febrile UTI in children [30]. Since that time several clinical studies have been conducted for assessing the diagnostic value of NGAL and KIM-1 for predicting UTI and APN in children. Most of them have demonstrated usefulness of uNGAL for early diagnosis of UTI in children [2, 11, 12, 14, 18, 31], for differentiation of UTI from contamination and colonization $[32,33]$, and for monitoring of treatment response [6, 14, 34]. In our previous study (2018), we found that UNGAL had lower sensitivity and specificity than serum NGAL for diagnosing febrile UTI as well as that UNGAL was not useful for diagnosing non-febrile UTI [21].

Few studies have assessed the diagnostic value of uNGAL for detecting APN in children, but the results are not clear. Arambasic et al. revealed high uNGAL sensitivity $(92.5 \%)$ and specificity $(91 \%)$ for differentiation of APN from other febrile infection. They also showed utility of uNGAL for differentiation of upper from lower UTI [24]. In a similar study, Nicavar et al. demonstrated smaller uNGAL sensitivity (76\%) and specificity (77\%) for distinguishing APN from other febrile infections [6]. Ghasemi et al. reported that UNGAL was not sensitive enough (67\%) for distinguishing APN from lower UTI, but it was a highly specific (98\%) marker. They concluded that uNGAL should not be used to predict renal parenchymal involvement in febrile UTI [26]. In contrast Piccoli et al. did not find any difference in uNGAL levels in adults with and without APN [35].

There are three unique studies that compare usefulness of UNGAL with uKIM-1 for predicting febrile UTI and APN in children. In a study by Yim et al., both normalized and non-normalized values of UNGAL have demonstrated higher sensitivity and specificity than those of UKIM-1 for diagnosing febrile UTI. Differentiation of the APN from lower UTI was only improved by uNGAL and uNGAL/ uCr (sensitivity $75 \%$ and $90 \%$, specificity $74 \%$ and $63 \%$, respectively) [14]. Lee et al. in a clinical study found also higher uNGAL/uCr sensitivity and specificity than uKIM$1 / \mathrm{uCr}$ for detecting febrile UTI. Only uNGAL/uCr was able to distinguish APN from lower UTI (sensitivity 63\%, specificity $86 \%$ ) [18]. Valdimarsson et al. reported very high uNGAL and uNGAL/uCr sensitivity and specificity for predicting febrile UTI, smaller for UKIM-1 and uKIM-1/ $\mathrm{uCr}$ [12].

Urbschat et al. performed a study in adults with UTI and found that neither uNGAL nor $\mathrm{uNGAL} / \mathrm{uCr}$ have reached significant differences between patients with upper and lower UTI. uKIM-1 levels were significantly elevated in patients with upper UTI compared with lower UTI. However, in authors' opinion these levels were close to the detection limit, and were too low for reliable differentiation between both groups. In this research diag- nosis of upper and lower UTI was based only on clinical and laboratory data [28]. It is well known that these data allow for diagnosis of upper UTI, but are unable to identify parenchymal involvement from "pyelitis". According to the reports outlined above, uNGAL and uNGAL/uCr demonstrated better sensitivity for diagnosing febrile UTI in children than for differentiation of APN from lower UTI. uKIM-1 and UKIM-1/uCr showed small sensitivity for predicting febrile UTI and were not useful for diagnosing APN.

In clinical practice fever is commonly used for differentiation of APN from lower UTI in children. However, the incidence of renal parenchymal involvement in children with febrile UTI varies from $16.3 \%$ to $67 \%$ [4, 16 , $26,36]$. In our study $44 \%$ of children with febrile UTI presented abnormal DMSA scan. Few authors revealed that renal parenchymal involvement in UTI is related to longer duration of fever before antibiotic treatment $[9,17$, 36]. In the present study, similar to Yun et al. and Seo et al. reports, no significant differences in duration of fever before antibiotic treatment were found between children with APN and lower UTI $[4,5]$.

Numerous studies evaluated usefulness of inflammatory markers for predicting APN. Commonly used laboratory parameters such as WBC and CRP have low sensitivity and specificity for detecting APN, and they are not recommended for differentiation of APN from lower UTI in children [7]. The role of PCT in APN diagnosis is not clear. Metaanalysis by Zhang et al. demonstrated that PCT cut-off value of $1.0 \mathrm{ng} / \mathrm{ml}$ can be useful for predicting APN in young children, but further multicenter studies are needed to validate this cut-off value of PCT for diagnosing APN [37]. In contrast, Ghasemi et al. showed better sensitivity for WBC and erythrocyte sedimentation rate (ESR) than for UNGAL for diagnosis APN [26]. In the present study we also found that PCT and CRP were better diagnostic markers of APN in children than UNGAL and UKIM-1. While conventional inflammatory markers increase in many clinical settings, the utility of their elevated values in predicting APN in febrile children is still under debate [14].

In healthy individuals, NGAL is expressed at very low levels in neutrophils and epithelial cells of several tissues including the kidney, liver, lung and colon [26, 27]. Its expression increased in the presence of epithelial injury and inflammation [12]. In AKI, NGAL is massively upregulated in the thick ascending limb of Henle's loop, distal tubule and collecting duct and released into blood and urine [13, 14]. More severe forms of APN can provide to acute tubule-interstitial kidney injury [8, 27, 34]. However, several investigators demonstrated increased levels of uNGAL in patients with APN, who had no specific signs or symptoms of AKI [14, 28]. It is caused by an increased release from activated neutrophils in the infected kidney to prevent bacterial iron uptake and growth [14, 18, 34], and from leukocytes present in the urine and urothelium 
[28]. This notion is supported by the findings that a high level of uNGAL could be detected in patients with APN and lower UTI [14, 28], as well as by an observation that uNGAL levels significantly decrease following antibiotic treatment [6, 14, 17, 34], and by positive correlations of uNGAL with inflammatory markers and pyuria $[6,14,18$, 38]. Petrovic et al. demonstrated significantly higher levels of uNGAL in children with longer duration of inflammation than those with shorter duration [34]. We found positive correlations of uNGAL with pyuria and of uNGAL/ uCr with uKIM-1/uCr.

In healthy population KIM-1 is released at a very low level from proximal tubules [18]. The large pediatric reference range study performed by Zwiers et al. in healthy children aged 1 day to 1 year, has demonstrated extremely low levels of uKIM-1 in almost all children, and no relation of uKIM-1 with age and gender [39]. McWilliam et al. found also diurnal variation of uKIM-1 levels, with higher values in the morning, lower in the evening [40]. In AKI, uKIM-1 is highly upregulated in proximal tubule cells and extracellular domain of KIM-1 is released into urine soon after tubule damage [18, 28, 34]. In patients with a severe form of APN, proximal tubule is not mainly affected [28]. Since KIM-1 represents a marker of proximal tubule injury, the low levels of UKIM-1 in patients with APN are not unexpected [14, 18, 28]. The biological role of NGAL and KIM-1 in AKI is still under debate. It is speculated that both biomarkers are expressed in order to aid regeneration after kidney damage. They induce reepithelization and reduce apoptosis $[3,17,31,34]$.

There were some limitations of our study. This was a single-center study, with a small cohort of children and absence of data controls. Small sample size may have contributed to results of our study. Statistical results could be improved after recruiting more patients. Our urine samples for biomarkers assessment were collected at varying times of the day. It could also affect results of uKIM-1 levels.

\section{Conclusions}

This study was performed to evaluate the potential value of two urinary biomarkers of AKI, uNGAL and uKIM-1, for prediction APN in children with febrile UTI. The results of this prospective study show that among children aged 1-24 months, uNGAL and uKIM-1 were not effective diagnostic markers for APN. Both these markers cannot be used in clinical practice to differentiate APN from lower UTI.

\section{The authors declare no conflict of interest.}

\section{References}

1. Subcommittee on Urinary Tract Infection, Steering Committee on Quality Improvement and Management, Roberts KB
(2011): Urinary tract infection: Clinical practice guideline for the diagnosis and management of the initial UTI in febrile infants and children 2 to 24 months. Pediatrics 128: 595-610.

2. Lubell TR, Barasch JM, Xu K, et al. (2017): Urinary neutrophil gelatinase-associated lipocalin for the diagnosis of urinary tract infections. Pediatrics 140: e20171090.

3. Wang J-J, Chi N-H, Huang T-M, et al. (2018): Urinary biomarkers predict advanced acute kidney injury after cardiovascular surgery. Critical Care 22: 108.

4. Yun BA, Yang EM, Kim CJ (2018): Plasma neutrophil gelatinase-associated lipocalin as a predictor of renal parenchymal involvement in infants with febrile urinary tract infection: a preliminary study. Ann Lab Med 38: 425-430.

5. Seo WH, Nam SW, Lee EH, et al. (2014): A rapid plasma neutrophil gelatinase-associated lipocalin assay for diagnosis of acute pyelonephritis in infants with acute febrile urinary tract infections: a preliminary study. Eur J Pediatr 173: 229-232.

6. Nickavar A, Safaeian B, Valavi E, et al. (2016): Validity of neutrophil gelatinase associated lipocaline as a biomarker for diagnosis of children with acute pyelonephritis. Pediatr Urol 13: 2860-2863.

7. Shaikh N, Borrell JL, Evron J, et al. (2015): Procalcitonin, C-reactive protein, and erythrocyte sedimentation rate for the diagnosis of acute pyelonephritis in children. Cochrane Database Syst Rev 1: CD009185.

8. Rafiei A, Mohammadjafari H, Bazi S, et al. (2015): Urinary neutrophil gelatinase-associated lipocalin (NGAL) might be an independent marker for anticipating scar formation in children with acute pyelonephritis. J Renal Inj Prev 4: 39-44.

9. Karavanaki KA, Soldatou A, Koufadaki AM, et al. (2017): Delayed treatment of the first febrile urinary tract infection in early childhood increased the risk of renal scarring. Acta Pediatr 106: 149-154.

10. Krzemień G, Szmigielska A, Turczyn A, et al. (2016): Urine interleukin-6, interleukin-8 and transforming growth factor $\beta 1$ in infants with urinary tract infection and asymptomatic bacteriuria. Centr Eur J Immunol 41: 260-267.

11. Jung N, Byun HJ, Park JH, et al. (2018): Diagnostic accuracy of urinary biomarkers in infants younger than 3 months with urinary tract infection. Korean J Pediatr 61: 24-29.

12. Valdimarsson S, Jodal U, BarregÍrd L, et al. (2017): Urine neutrophil gelatinase-associated lipocalin and other biomarkers in infants with urinary tract infection and in febrile controls. Pediatr Nephrol 32: 2079-2087.

13. Singer E, Markó L, Paragas N, et al. (2013): Neutrophil gelatinase-associated lipocalin: pathophysiology and clinical applications. Acta Physiol (Oxf) 207: 663-672.

14. Yim HE, Yim H, Bae ES, et al. (2014): Predictive value of urinary and serum biomarkers in young children with febrile urinary tract infections. Pediatr Nephrol 29: 2181-2189.

15. Nasioudis D, Witkin SS (2015): Neutrophil gelatinaseassociated lipocalin and innate immune responses to bacterial infections. Med Microbiol Immunol 204: 471-479.

16. Forster DS, Johnson K, Patel V, et al. (2017): Urinary NGAL deficiency in reccurent urinary tract infections. Pediatr Nephrol 32:1077-1080.

17. Sim JH, Yim HE, Choi BM, et al. (2015): Plasma neutrophil gelatinase-associated lipocalin predicts acute pyelonephritis in children with urinary tract infections. Pediatric Research 78: 48-55.

18. Lee H-E, Kim DK, Kang HK, et al. (2015): The diagnosis of febrile urinary tract infection in children may be facilitated by urinary biomarkers. Pediatr Nephrol 30: 123-130. 
19. Filho LT, Grande AJ, Colonetti T, et al. (2017): Accuracy of neutrophil gelatinase-associated lipocalin for acute kidney injury diagnosis in children: systematic review and metaanalysis. Pediatr Nephrol 32: 1979-1988.

20. Kisiel A, Roszkowska-Blaim M, Pańczyk-Tomaszewska M, et al. (2017): Effect of perinatal risk factors on neutrophil gelatinase-associated lipocalin (NGAL) level in umbilical and peripheral blood in neonates. Cent Eur J Immunol 3: 274-280.

21. Krzemień G, Pańczyk-Tomaszewska, Adamczuk D, et al. (2018): Neutrophil gelatinase-associatedl lipocalin: a biomarker for early diagnosis of urinary tract infections in infants. Advs Exp Med Biol 38: 71-80.

22. Forster CS, Davarajan P (2017): Neutrophil GelatinaseAssociated Lipocalin: Utility In Urologic Conditions. Pediatr Nephrol 32: 377-381.

23. Price JR, Guran L, Lim JY, et al. (2017): Neutrophil gelatinase-associated lipocalin biomarker and urinary tract infections: a diagnostic case-control study (NUTI Study). Female Pelvic Med Reconstr Surg 23: 101-107.

24. Arambašic J, Mandić S, Debeliak Ž, et al. (2016): Differentiation of acute pyelonephritis from other febrile states in children using urinary neutrophil gelatinase-associated lipocalin (uNGAL). Clin Chem Lab Med 54: 55-61.

25. Kim BK, Yim HE, Yoo KH (2017): Plasma neutrophil gelatinase-associated lipocalin: a marker of acute pyelonephritis in children. Pediatr Nephrol 32: 477-484.

26. Ghasemi K, Esteghamati M, Borzoo S, et al. (2016): Predictive accuracy of urinary neutrophil gelatinase associated lipocalin (NGAL) for renal parenchymal involvement in children with acute pyelonephritis. Electron Physician 8: 1911-1917.

27. Kim BH, Yu N, Kim HR, et al. (2014): Evaluation of the optimal neutrophil gelatinase-associated lipocalin value as a screening biomarker for urinary tract infections in children. Ann Lab Med 34: 354-359.

28. Urbschat A, Obermüller N, Paulus P, et al. (2014): Upper and lower urinary tract infections can be detected early but not be discriminated by urinary NGAL in adults. Int Urol Nephrol 46: 2243-2249.

29. Ichino M, Kuroyanagi Y, Kusaka M, et al. (2009): Increased urinary neutrophil gelatinase associated lipocalin levels in a rat model of upper urinary tract infection. J Urol 181: 23262331.

30. Lee H-E, Lee SH, Baek M, et al. (2013): Urinary measurement of neutrophil gelatinase associated lipocalin and kidney injury molecule-1 helps diagnose acute pyelonephritis in a practical model. J Biomarkers 2013: 413853.

31. Ylmaz A, Sevketoglu E, Gedikbasi A, et al. (2009): Early prediction of urinary tract infection with urinary neutrophil gelatinase associated lipocalin. Pediatr Nephrol 24: 23872392.

32. Hatipoglu S, Sevketoglu E, Gedikbasi A, et al. (2011): Urinary MMP-9/NGAL complex in children with acute cystitis. Pediatr Nephrol 26: 1263-1268.

33. Forster CS, Jackson E, Ma Q, et al. (2018): Predictive ability of NGAL in identifying urinary tract infection in children with neurogenic bladders. Pediatr Nephrol 33: 1365-1374.

34. Petrovic S, Bogavac-Stanojevic N, Peco-Antic A, et al. (2013): Clinical application neutrophil gelatinase-associated lipocalin and kidney injury molecule- 1 as indicators of inflammation persistence and acute kidney injury in children with urinary tract infection. Biomed Res Int 2013: 947157.

35. Piccoli GB, Ferraresi M, Aroasio E, et al. (2012): The search for perfect biomarkers in acute kidney damage: the case of
NGAL from AKI to acute pyelonephritis: back to the clinic? Nephrol Dial Transplant 2: 3665-3666.

36. Printza N, Farmaki E, Piretzi K, et al. (2012): Acute phase $99 \mathrm{mTc}$-dimercaptosuccinic acid scan in infants with first episode of febrile urinary tract infection. World J Pediatr 8: 52-56.

37. Zhang H, Yang, Lin L, et al. (2016): Diagnostic value of serum procalcitonin for acute pyelonephritis in infants and children with urinary tract infections: an updated meta-analysis. World J Urol 34: 431-441.

38. Decavele AS, Dhondt L, De Buyzere ML, et al. (2011): Increased urinary neutrophil gelatinase associated lipocalin in urinary tract infection and leukocyturia. Clin Chem Lab Med 49: 999-1003.

39. Zwiers AJ, deWildt SN, de Rijke YB, et al. (2015): Reference intervals for renal injury biomarkers neutrophil gelatinaseassociated lipocalin and kidney injury molecule-1 in young infants. Clin Chem Lab Med 53: 1279-1289.

40. McWilliam SJ, Antoine DJ, Sabbisetti V, et al. (2014): Reference intervals for urinary renal injury biomarkers KIM-1 and NGAL in healthy children. Biomark Med 8: 1189-1197. 